\title{
Factors affecting implementation and pass rates of surgical instrument moistening
}

\author{
Yongdeng Huang ${ }^{1,2}$, Yan Huang ${ }^{1,2}$, Yanhua Chen ${ }^{1,2}$, Wei Pan ${ }^{1,2}$, Juan $\mathrm{Hu}^{1,2^{*}}$ and Liangying $\mathrm{Yi}^{1,2^{*}}$
}

\begin{abstract}
Background: Moistening of surgical instruments affects the quality of instrument cleaning, thereby affecting the degree of cross-contamination and in-hospital infection among patients. Surgical instruments should be kept moist immediately after use in order to avoid concentrations of contamination remaining on surgical instrument surfaces. Implementation and pass rates of surgical instrument moistening have been rarely studied. We aimed to investigate the factors affecting implementation and pass rates of surgical instrument moistening.

Methods: A cross-sectional study was conducted to investigate surgical instrument moistening procedures within 22 clinical departments of the West China Second University Hospital, Sichuan University over 122 days between September and December 2019. We collected data from departmental staff using an interviewer-administrated questionnaire. Data about implementation and pass rates of surgical instrument moistening was analyzed in SPSS20.0.

Results: Implementation and pass rates of surgical instrument moistening were $57.25 \%$ and $31.98 \%$, respectively. Factor analysis showed that implementation rates of moistening were affected by instrument structure $\left(X^{2}=143.670\right.$; $P=0.001)$, the number of instruments inside the pack $\left(X^{2}=140.135 ; P=0.001\right)$, and the person responsible for keeping surgical instruments moist $\left(X^{2}=8.052 ; P=0.005\right)$. Correlation analysis showed that instrument structure and the number of instruments inside the pack were negatively correlated with implementation rates of moistening. The more complex the structure and the greater the number of the instruments inside the pack, the lower implementation rates of moistening.
\end{abstract}

Conclusion: Implementation and pass rates of surgical instrument moistening were low, and failed to meet the central sterile supply department applicable industrial standard, hence the potential risk of hospital-acquired infection was considerable. Staff that manipulate reusable surgical instruments should be trained to properly moisten the instruments and institutional protocols should be established to ensure standardization and respect of guidelines.

Keywords: Surgical instruments, Sterilization, Nurses, Cross infection

\section{Background}

Thorough cleaning of surgical instruments is vital to their disinfection and sterilization [1]. However, the efficiency and degree of sterilization depend to a large extent on logistics and sterilization management procedures,

\footnotetext{
*Correspondence: 944807470@qq.com; yiliangying88@163.com ${ }^{1}$ Central Sterile Supply Department, West China Second University Hospital, Sichuan University/West China School of Nursing, Sichuan University, Chengdu, Sichuan, China

Full list of author information is available at the end of the article
}

namely surgical instrument transportation, storage and cleaning procedures. There is the risk of surgical instruments not being cleaned properly or immediately after use, including insufficient moistening, which results in potentially hazardous concentrations of contamination remaining on surgical instrument surfaces, leading to cross-contamination. Incomplete cleaning of instruments is one form of poor sterilization which contributes to hospital-acquired infections. In a landmark study of sterilization procedures in the central sterile supply department (CSSD), Liu and Liang (2013) stated that surgical 
site infections accounted for 14-16\% of hospital-acquired infections among hospitalized patients, and that approximately $20 \%$ of surgical site infections were ascribed to poor instrument hygiene quality [2]. According to the Central Sterile Supply Department (CSSD)'s directive (transl.)-Part 2: Standard for Operating Procedure of Cleaning, Disinfection and Sterilization (WS 310.2-2016) [3], "The user shall timeously remove visible contaminants from diagnostic and treatment instruments, apparatus and articles after use, and moisten them according to actual situations."

There have been numerous studies investigating methods for keeping surgical instruments moist and effects of moistening, but factors affecting implementation and pass rates of surgical instrument moistening have been rarely studied. This case study considers the factors in relation to moistening of surgical instruments which affects the quality of instrument cleaning, and thereby affecting the degree of cross-contamination and in-hospital infection among patients, which should be monitored by medical staff and hospital management in their efforts to lower infection and contamination levels.

\section{Methods}

\section{Study setting}

A survey was conducted within 22 clinical departments of the West China Second University Hospital, Sichuan University over 122 days between September and December 2019. The 22 departments in the hospital rely on reusable surgical instruments; other departments in the hospital used disposable surgical instruments or did not use surgical instruments. The 22 departments have a total of 1580 hospital beds, and the average number of operations performed across the 22 departments per year is around 70,000 .

\section{Survey tools}

We designed an interviewer-administrated questionnaire based on the aforementioned CSSD directive (Part 2: Standard for Operating Procedure of Cleaning, Disinfection and Sterilization (WS 310.2-2016)) [3] in order to investigate instrument moistening procedures in the hospital. We pre-tested the questionnaire to assess its reliability and validity before using it to collect data. Cronbach's alpha for the questionnaire was 0.911 and test-retest reliability coefficient was 0.825 . The questionnaire which had high reliability and validity was composed of 4 parts:

Part 1: Consisted of 7 questions, and was used to collect data about which campus and which department each respondent worked in, as well as the respondent's name, age, length of service, educational background, and job title.
Part 2: Consisted of 4 open-ended questions regarding surgical procedure names, a description of the relevant surgical pack, the number of instruments inside the pack, the end date and end time of surgery, and time of moistening; these were followed by 11 closed-ended questions regarding place of moistening, the person responsible for moistening, moistening method, moisturizing liquid/ agent, and effects of moistening on different instruments.

Part 3 and Part 4: Each of these questionnaire parts contained 1 question. Each respondent was asked to detail reasons why moistening procedures might fail (12 possible answers), and (in Part 4) provide reasons why instruments were not moistened (7 possible answers). Each respondent was allowed to choose 1 or more answers to each of the 2 questions. However, the respondent was not required to answer the questions in Part 3 and 4 if instruments were moistened in compliance with the CSSD standard.

The questionnaires were administrated by us who had received training on helping the respondents fill in questionnaires and also possessed experience of conducting on-site investigations for keeping surgical instruments moist. We visited the 22 departments to investigate the following: whether surgical instruments were being kept moist after use; type of moistening agent used; place and time of moistening; method for keeping instruments moist; and, effects of moistening on different instruments. A sample of nurses and other hospital workers were asked to explain why moistening procedures might fail, and specify reasons why the instruments were not being kept moist.

We have classified reusable surgical instruments into three categories: common instruments, instruments with articulation joints or grooves, and cannulated instruments. We recorded information concerning the effects of moistening of instruments in different surgical packs, and calculated pass rates of moistening for the three categories of instruments and those for surgical packs containing different numbers of instruments. We also collected information concerning the effects of moistening handled by different persons (nurses or workers). We have supplemented the information provided by respondents by taking photos and recording videos demonstrating moistening procedures, for the purpose of further analysis and discussion by the research team.

Moistening is considered adequate when moisturizing agent is sprayed on the instruments immediately after use; when the surface, articulation joints and grooves of the instruments are fully and evenly covered with moisturizing gel; and when they are kept moist right up to the moment of delivery to CSSD for subsequent processing [4]. We found that Conbizyme instrument moisturizing gel is used in the hospital. Conbizyme is the brand name of the 
moisturizing gel produced by Nanjing Jusha Commercial \& Trading Co., Ltd. in China; it is a ready-to-use instrument moisturizing product which is sprayed directly without dilution on instruments at a distance of around $10 \mathrm{~cm}$.

Three are three spray methods: Random spray is defined as discretionary spray, the degree of which varies according to moistening handler's own convenience without regard to the effect of moistening. In the 'Evenly' spray method, the instrument surface is evenly covered with moisturizing agent in order to effectively moisten the instrument. With the Targeted spray method, the instruments with their different structures from different angles are sprayed in such a way to achieve complete moistening. We took notes of the respective spray methods during the on-site inspection.

All research methods were carried out in accordance with the relevant guidelines and regulations. This study was conducted in accordance with the Declaration of Helsinki, and was approved by the Medical Ethics Committee of West China Second University Hospital, Sichuan University (No.: YXKY2020LSP(171)). Informed consent for participation was obtained from all nurses and workers targeted by this study. During our on-site investigation, we presented our work-related identity cards to the nurses and workers of the 22 departments, orally explained the purpose and significance of this study to them, and informed them that they had the right to refuse to answer our questions or withdraw from the study at any time. We then allowed them to choose whether to participate in the study. After obtaining their verbal consent to participate in this study, we verbally presented them with the questionnaire items, and then recorded their responses to the questionnaire items. The whole interview process, including our oral presentation and obtaining participants' verbal consents, was witnessed by the head nurse of their respective department. The Medical Ethics Committee of West China Second University Hospital, Sichuan University reviewed and approved the research proposal and procedure of verbal consent of this study, and felt that we did not need to obtain written consent from the participants.

\section{Statistical methods}

We used MS Excel 2007 for data entry, and analyzed data using the SPSS20.0 statistical package. We applied the Pareto principle in our analysis of factors, i.e. whether the number of significant factors is greater than that of non-significant factors [5]. The data are expressed as percentages, frequency and relative frequency. In addition, the Chi-square test and rank sum test are used to compare factors, and the degree of correlation between variables has been analyzed by applying the rank correlation approach. Bivariable test results show factors affecting pass rates of moistening to be statistically significant at the $(P<0.05)$ level.

\section{Results \\ Implementation of surgical instrument moistening procedures}

It was found that only reusable surgical instruments from 3 (13.64\%) of the 22 departments were moistened. A total of 45,717 reusable surgical instruments from the 22 departments were investigated, among which 26,172 instruments (implementation rate $=57.25 \%$ ) were kept moist, and 8369 instruments (pass rate $=31.98 \%$ ) were moistened in compliance with the CSSD standard. The implementation and pass rates of surgical instrument moistening were low, as shown in Table 1.

\section{Reasons why surgical instruments were not being kept moist}

The Pareto diagram has been utilized to analyze the reasons why surgical instruments were not being kept moist. Our analysis showed that the main reasons included "did not know that instruments must be moistened immediately after use", "did not know how to moisten instruments", and "did not know the importance of keeping instruments moist", as shown in Fig. 1.

\section{Reasons why moistening procedures failed or were insufficient}

The Pareto diagram has been adopted in order to analyze the reasons behind failures in moistening procedures or coverage. Reasons include improper time for moistening, and lack of standard operating procedures for keeping instruments moist, as shown in Fig. 2.

\section{Time of moistening (interval between end of instrument use and start of moistening)}

Among the three departments which moistened instruments, the interval between end of instrument use and start of moistening among the outpatient operating rooms lasted $4.04 \mathrm{~min}$ on average $(4.001<95 \%$ confidence interval $<4.079)$; that of the inpatient operating rooms lasted $4.88 \mathrm{~min}$ on average $(4.862<95 \%$ confidence interval $<4.898)$; and, that of the radiology department lasted $172.5 \mathrm{~min}$ on average $(172.169<95 \%$ confidence interval $<172.831$ ).

\section{Spraying methods}

It was found that the percentages of instruments subject to random spray, evenly spray, and targeted spray were $83.56 \%, 12.33 \%$, and $4.11 \%$, respectively. 
Table 1 Implementation of surgical instrument moistening in each department

\begin{tabular}{llllll}
\hline Departments & $\begin{array}{l}\text { Number } \\
\text { of surgical } \\
\text { instruments (\%) }\end{array}$ & $\begin{array}{l}\text { Number of } \\
\text { instruments kept } \\
\text { moist }\end{array}$ & $\begin{array}{l}\text { Implementation } \\
\text { rate of moistening } \\
(\%)\end{array}$ & $\begin{array}{l}\text { Number of instruments moistened } \\
\text { in compliance with CSSD standard }\end{array}$ & $\begin{array}{l}\text { Pass rate of } \\
\text { moistening } \\
\text { (\%) }\end{array}$ \\
\hline Inpatient operating rooms & $27,105(59.29)$ & 21,506 & 79.34 & 6139 & 28.55 \\
Delivery rooms & $12,611(27.59)$ & 0 & 0 & - & - \\
Outpatient operating rooms & $4097(8.96)$ & 4097 & 100 & 2152 & - \\
In vitro fertilisation & $818(1.79)$ & 0 & 0 & - & - \\
Pediatric Cardiology & $328(0.72)$ & 0 & 0 & 78 & - \\
Radiology & $569(1.24)$ & 569 & 100 & - & 3.71 \\
Other clinical departments & $189(0.41)$ & 0 & 0 & 8369 & 31.98 \\
Total & 45,717 & 26,172 & 57.25 & - \\
\hline
\end{tabular}

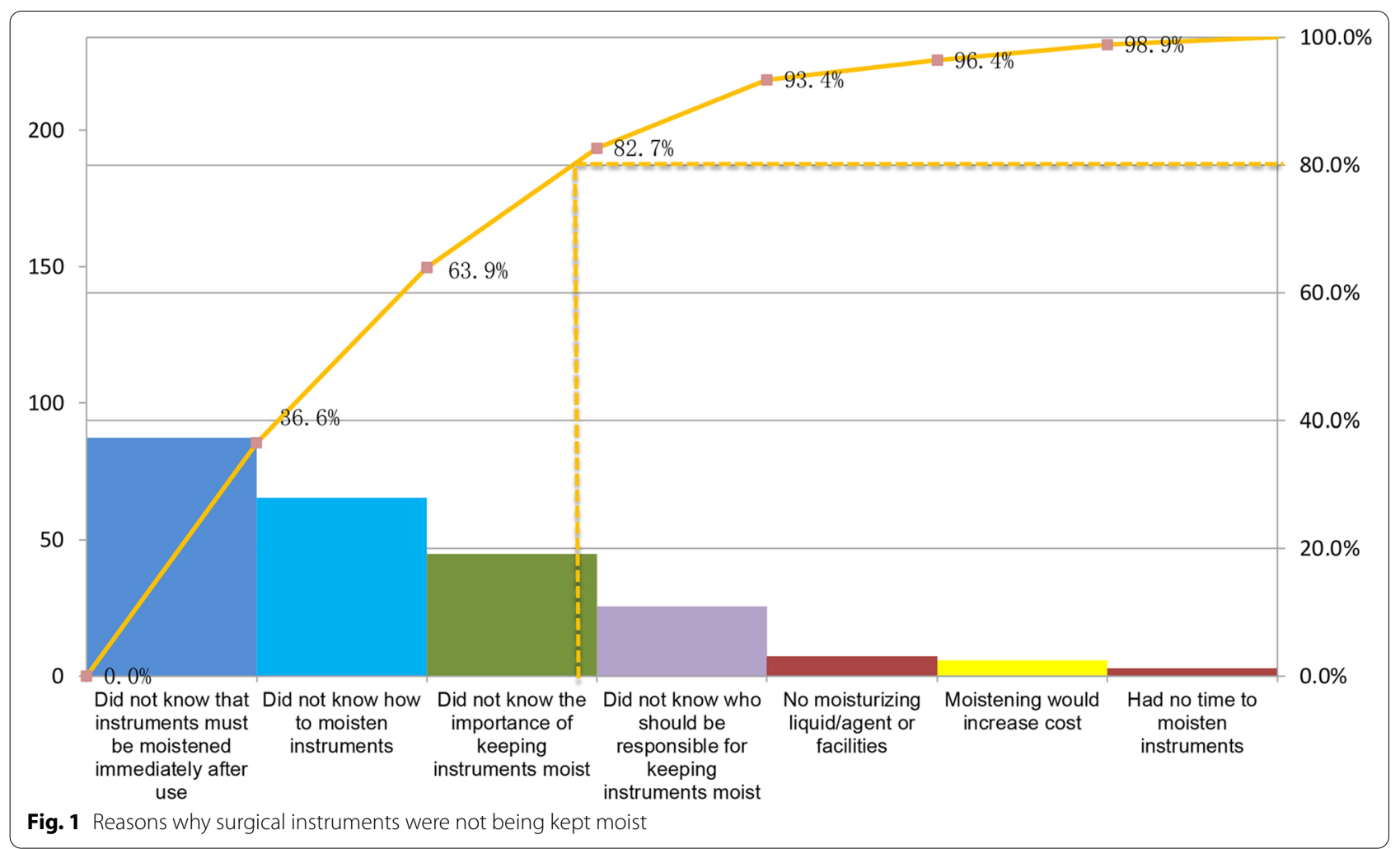

\section{Factors affecting keeping surgical instruments moist}

Pass rates of moistening were calculated for instruments with different structures, surgical packs containing different numbers of instruments, and instruments moistened by different staff members. Single factor analysis was conducted using the Chi-square test, the results of which show that instrument structure, the number of instruments inside the pack, and the person responsible for keeping instruments moist, constitute the main factors affecting the pass rates, as shown in Table 2.

Correlation analysis was conducted for the factors affecting moistening of surgical instruments. The results show that the more complex the structure and the greater the number of the instruments inside the pack, the lower the pass rates of moistening; the results also show that the pass rates among nurses was higher than that among workers, as detailed in Table 3.

\section{Discussion}

This study shows that the implementation rate for the sample of surgical instruments in the hospital was only $57.25 \%$. The main reasons for this were that many of the nurses and workers of the clinical departments were not fully knowledgeable of the methods or importance of 


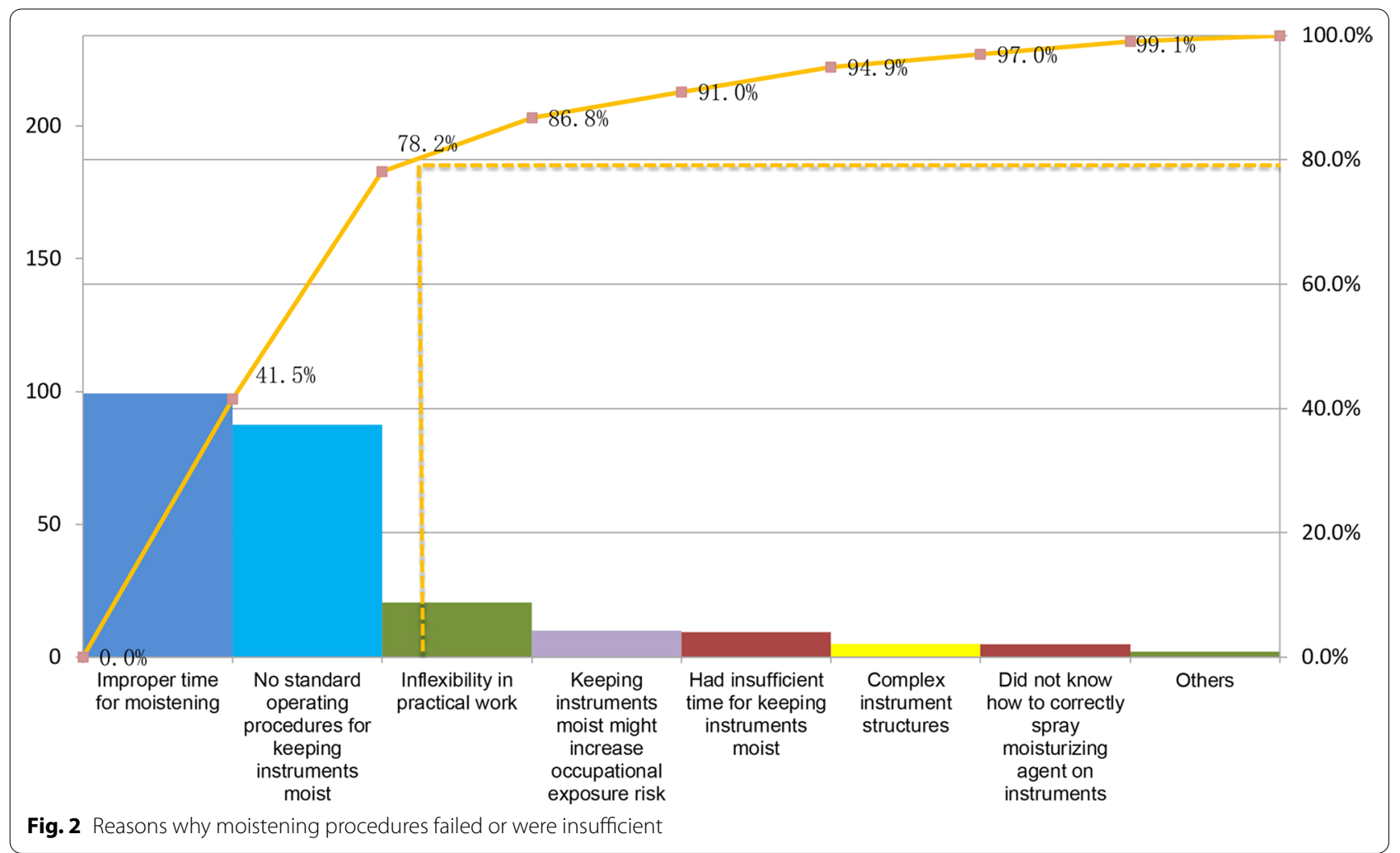

keeping surgical instruments moist. It is evident that many of the nurses and workers are in need of more training as regards to keeping surgical instruments moist and reducing the risk of cross-contamination. The low pass rates are also a reflection of a lack of standard operating procedures for keeping instruments moist, and the need for harmonization of guidelines. As reported by other scholars in the field, contaminants should be removed from the reusable surgical instruments as soon as possible following use and sent to CSSD within $30 \mathrm{~min}$ in order to minimize the risk of cross-contamination; in short, the instruments should be kept moist [6]. Numerous dried contaminants on the instruments can be difficult to remove, will increase the cost of cleaning, and might even cause corrosion, which

Table 2 Single factor analysis on factors affecting keeping surgical instruments moist

\begin{tabular}{|c|c|c|c|c|c|}
\hline Item & $\begin{array}{l}\text { Number of } \\
\text { instruments kept } \\
\text { moist (\%) }\end{array}$ & $\begin{array}{l}\text { Number of instruments moistened } \\
\text { in compliance with CSSD standard }\end{array}$ & $\begin{array}{l}\text { Pass rate of } \\
\text { moistening } \\
(\%)\end{array}$ & $x^{2}$ & $P$ value \\
\hline Instrument structure & & & & 143.670 & 0.001 \\
\hline Common instruments & $7852(30.00)$ & 4776 & 60.83 & & \\
\hline Instruments with articulation joints or grooves & $15,703(60.00)$ & 3305 & 21.05 & & \\
\hline Cannulated instruments & $2617(10.00)$ & 288 & 1.10 & & \\
\hline The number of instruments inside the pack & & & & 140.135 & 0.001 \\
\hline$<10$ pcs & $1581(6.04)$ & 1316 & 83.24 & & \\
\hline $10-20$ pcs & $2577(9.85)$ & 1733 & 67.25 & & \\
\hline $21-30$ pcs & $2018(7.71)$ & 875 & 43.36 & & \\
\hline $31-40$ pcs & $781(2.98)$ & 174 & 22.28 & & \\
\hline$>40$ pcs & $250(0.96)$ & 33 & 13.20 & & \\
\hline Person responsible for keeping instruments moist & & & & 8.052 & 0.005 \\
\hline Nurses & $18,320(70.00)$ & 7402 & 40.40 & & \\
\hline Workers & $7852(30.00)$ & 967 & 12.32 & & \\
\hline
\end{tabular}


Table 3 Correlation analysis on factors affecting keeping surgical instruments moist

\begin{tabular}{|c|c|c|}
\hline Item & Correlation coefficient & $P$ value \\
\hline Instrument structure & -0.525 & 0.001 \\
\hline $\begin{array}{l}\text { The number of instruments inside } \\
\text { the pack }\end{array}$ & -0.203 & 0.001 \\
\hline $\begin{array}{l}\text { Person responsible for keeping } \\
\text { instruments moist }\end{array}$ & 0.194 & 0.001 \\
\hline
\end{tabular}

impairs instrument function and shorten the service life of instruments, and thus increases the average unit cost [4]. Therefore, surgical instruments should be kept moist immediately after use if they are unavailable for timely cleaning. It has been demonstrated in some studies that contaminants would dry within several minutes if not processed immediately after use [7]; bacteria could multiply in 4-20 min on dried contaminants, forming a biofilm within $2 \mathrm{~h}$ [8]. The earlier surgical instruments are moistened, the better the moistening effect is. It is therefore recommended that surgical instruments should be kept moist immediately following surgery [9].

According to the correlation analysis, the main factors influencing the moistening of surgical instruments include instrument structure, the number of instruments inside the pack, and the person responsible for keeping instruments moist. The more complex the instrument structure, the less effective the moistening; therefore, special methods should be used for instruments with more complex structures. Ultrasonic scalpels, connecting cables for argon beam coagulation, among other instruments with complex structures, should be kept moist via separate multienzyme soaking [10]. Appropriate spraying methods should be adopted in order to ensure sufficient moistening, e.g. unlock instrument articulation joints prior to spraying, and carrying out targeted spray on articulation joints, grooves and cannulations of instruments [9], and place instruments in the recycling box for spraying when there are many instruments inside the pack. Pass rates among workers were significantly lower than those among the nurses, possibly because the workers' primary responsibility was to collect and deliver contaminated instruments, and because most of them only had secondary education qualifications, and lacked medical knowledge concerning the importance of moistening. Ideally, surgical instruments should be moistened by nurses as they have more professional knowledge and higher sense of responsibility.

\section{Conclusion}

It was found in this case study that implementation and pass rates of surgical instrument moistening were low, and failed to meet the CSSD applicable industrial standard, hence the potential risk of hospital-acquired infection was considerable. Staff that manipulate reusable surgical instruments should be trained to properly moisten the instruments and institutional protocols should be established to ensure standardization and respect of guidelines.

\section{Abbreviation}

CSSD: Central sterile supply department.

\section{Acknowledgements}

The authors would like to thank the nurses and workers working in the 22 clinical departments of the West China Second University Hospital, Sichuan University.

\section{Authors' contributions}

$\mathrm{YH}, \mathrm{JH}$ and $\mathrm{LY}$ contributed to the questionnaire design. All the authors carried out on-site investigation and data collection. $\mathrm{YH}$ and $\mathrm{YH}$ conducted data analysis. YH drafted the manuscript. JH, YC and WP revised the manuscript. All the authors read and approved the final manuscript.

\section{Funding}

This study was supported by Sichuan Provincial Health Department (No. 100374). The founder was not involved in the questionnaire design, on-site investigation, data collection, data analysis or preparation of the manuscript.

\section{Availability of data and materials}

The datasets used and/or analyzed during the current study are available from the corresponding authors on reasonable request.

\section{Declarations}

\section{Ethics approval and consent to participate}

This study was conducted in accordance with the Declaration of Helsinki, and this study was approved by the Medical Ethics Committee of West China Second University Hospital, Sichuan University (No.: YXKY2020LSP(171)). Verbal consent to participate in this study was obtained from all participants. The Medical Ethics Committee of West China Second University Hospital, Sichuan University reviewed and approved the research proposal and procedure of verbal consent of this study, and felt that it was not necessary to obtain written consent from participants. All data collected were confidential and used only by this study.

\section{Consent for publication}

Not applicable.

\section{Competing interests}

The authors declare that they have no competing interests.

\section{Author details}

${ }^{1}$ Central Sterile Supply Department, West China Second University Hospital, Sichuan University/West China School of Nursing, Sichuan University, Chengdu, Sichuan, China. ${ }^{2}$ Key Laboratory of Birth Defects and Related Diseases of Women and Children (Sichuan University), Ministry of Education, Chengdu, Sichuan, China.

Received: 12 June 2021 Accepted: 12 July 2021

Published online: 04 August 2021 


\section{References}

1. Zhou J, Cui Z. Comparison of cleaning quality and efficiency among three instrument pre-cleaning methods. Chin J Disinfect. 2016;33(8):798-9.

2. Liu Y, Liang M. On-the-job training in hospital central sterile supply department. Beijing: People's Military Medical Press; 2013.

3. National Health and Family Planning Commission of the People's Republic of China. Central Sterile Supply Department (CSSD)'s directive (transl.) - part 2: standard for operating procedure of cleaning, disinfection and sterilization (WS 310.2-2016); 2016. http://www.nhc.gov.cn/ewebe ditor/uploadfile/2017/01/20170105090606684.pdf. Accessed 27 Nov 2020.

4. Yao Y, Yu Z, Peng S, Zheng X, Wei H, Wang Y. Observation of cleaning effect of keeping surgical instruments moist overnight. Chin J Disinfect. 2017;34(6):576-7.

5. Koch R. The 80/20 principle. Trans Li H. Beijing: Haichao Press; 2001. pp. 3-16.

6. Nancy Chobin RN, AAS, ACSP, CSPM, CFER. Surgical instrument decontamination: a multistep process. AORN J. 2019:110(3):253-62.
7. Evangelista SS, Guimaraes NR, Garcia NB, Santos SG, Oliveria AC. Effectiveness of manual versus automated cleaning on Staphylococcus epidermidis biofilm removal from the surface of surgical instruments. Am J Infect Control. 2020;48(3):267-74.

8. Pacific Northwest National Laboratory. Just how fast can bacteria grow? It depends. Proteomics data validate model of bacteria growth. https:// www.pnnl.gov/science/highlights/highlight.asp?id=879. Accessed 1 May 2019

9. Guideline for cleaning and care of surgical instruments. In: Guidelines for perioperative practice. Denver, CO: AORN, Inc; 2019. pp. 401-440.

10. Li S, Yang J. Effect of two methods of keeping instruments moist for uterine cavity tissue suction tube cleaning. Chin J Disinfect. 2015;32(4):405-6.

\section{Publisher's Note}

Springer Nature remains neutral with regard to jurisdictional claims in published maps and institutional affiliations.
Ready to submit your research? Choose BMC and benefit from:

- fast, convenient online submission

- thorough peer review by experienced researchers in your field

- rapid publication on acceptance

- support for research data, including large and complex data types

- gold Open Access which fosters wider collaboration and increased citations

- maximum visibility for your research: over $100 \mathrm{M}$ website views per year

At $\mathrm{BMC}$, research is always in progress.

Learn more biomedcentral.com/submissions 